\title{
The absolute order on the hyperoctahedral group
}

Myrto Kallipoliti

Department of Mathematics

University of Athens

Panepistimioupolis 15784 Athens, Greece

mirtokemath.uoa.gr

\begin{abstract}
The absolute order on the hyperoctahedral group $B_{n}$ is investigated. It is shown that every closed interval in this order is shellable, those closed intervals which are lattices are characterized and their zeta polynomials are computed. Moreover, using the notion of strong constructibility, it is proved that the order ideal generated by the Coxeter elements of $B_{n}$ is homotopy Cohen-Macaulay and the Euler characteristic of the order complex of the proper part of this ideal is computed. Finally, an example of a non Cohen-Macaulay closed interval in the absolute order on the group $D_{4}$ is given and the closed intervals of $D_{n}$ which are lattices are characterized.
\end{abstract}

Résumé. Nous étudions l'ordre absolu sur le groupe hyperoctahédral $B_{n}$. Nous montrons que chaque intervalle fermé de cet ordre est shellable, caractérisons les treillis parmi ces intervalles et calculons les polynômes zêta de ces derniers. De plus, en utilisant la notion de constructibilité forte, nous prouvons que l'idéal engendré par les éléments de Coxeter de $B_{n}$ est Cohen-Macaulay pour l'homotopie, et nous calculons la caractéristique d'Euler du complexe associé à cet idéal. Pour finir, nous exhibons un exemple d'intervalle fermé non Cohen-Macaulay dans l'ordre absolu du groupe $D_{4}$, et caractérisons les intervalles fermés de $D_{n}$ qui sont des treillis.

Keywords: Coxeter group, hyperoctaherdal group, absolute order, Cohen-Macaulay poset, shellability

\section{Introduction and results}

Coxeter groups are fundamental combinatorial structures which appear in several areas of mathematics. Partial orders on Coxeter groups often provide an important tool for understanding the questions of interest. Examples of such partial orders are the Bruhat order and the weak order. We refer the reader to [7, 10, 15] for background on Coxeter groups and their orderings.

In this work we study the absolute order. Let $W$ be a finite Coxeter group with respect to the set $\mathcal{T}$ of all reflections in $W$. The absolute order on $W$ is denoted by $\operatorname{Abs}(W)$ and defined as the partial order on $W$ whose Hasse diagram is obtained from the Cayley graph of $W$ with respect to $\mathcal{T}$ by directing its edges away from the identity (see Section 2.1 for a precise definition). The poset $\operatorname{Abs}(W)$ is locally self-dual

\footnotetext{
${ }^{\dagger}$ The present research will be part of the author's Doctoral Dissertation at the University of Athens 
and graded. It has a minimum element, the identity $e \in W$, but will typically not have a maximum, since every Coxeter element of $W$ is a maximal element of $\operatorname{Abs}(W)$. Its rank function is called the absolute length and is denoted by $\ell_{\mathcal{T}}$. The absolute length and order arise naturally in combinatorics [2], group theory [5, 11], statistics [13] and invariant theory [15]. For instance, $\ell_{\mathcal{T}}(w)$ can also be defined as the codimension of the fixed space of $w$, when $W$ acts faithfully as a group generated by orthogonal reflections on a vector space $V$ by its standard geometric representation. In this case, the rank generating polynomial of $\operatorname{Abs}(W)$ satisfies

$$
\sum_{w \in W} t^{\ell_{\mathcal{T}}(w)}=\prod_{i=1}^{\ell}\left(1+e_{i} t\right),
$$

where $e_{1}, e_{2}, \ldots, e_{\ell}$ are the exponents [15, Section 3.20] of $W$ and $\ell$ is its rank. We refer to [2, Section 2.4] and [4, Section 1] for further discussion of the importance of the absolute order and related historical remarks.

In this paper we will be interested in the combinatorics and topology of $\operatorname{Abs}(W)$. These have been studied extensively for the interval $[e, c]:=N C(W, c)$ of $\mathrm{Abs}(W)$, known as the poset of noncrossing partitions associated to $W$, where $c \in W$ denotes a Coxeter element. For instance, it was shown in [3] that $N C(W, c)$ is shellable for every finite Coxeter group $W$. In particular, $N C(W, c)$ is homotopy CohenMacaulay and the order complex of $N C(W, c) \backslash\{e, c\}$ has the homotopy type of a wedge of spheres. The problem to determine the topology of the poset $\operatorname{Abs}(W) \backslash\{e\}$ and to decide whether $\operatorname{Abs}(W)$ is Cohen-Macaulay or shellable, was naturally posed by Athanasiadis (unpublished) and Reiner [1, Problem 3.1], see also [19, Problem 3.3.7]. Computer calculations carried out by Reiner showed that the absolute order is not Cohen-Macaulay for the group $D_{4}$. In the case of the symmetric group, it is still not known whether $\operatorname{Abs}\left(S_{n}\right)$ is shellable. However, the following result was obtained in [4].

[4. Theorem 1.1] The poset Abs $\left(S_{n}\right)$ is homotopy Cohen-Macaulay for all $n \geq 1$. In particular, the order complex of $\operatorname{Abs}\left(S_{n}\right) \backslash\{e\}$ is homotopy equivalent to a wedge of $(n-2)$-dimensional spheres and Cohen-Macaulay over $\mathbb{Z}$.

Here we focus on the hyperoctahedral group $B_{n}$. Contrary to the case of the symmetric group, not every maximal element of the absolute order on $B_{n}$ is a Coxeter element. The maximal intervals in $\operatorname{Abs}\left(B_{n}\right)$ include the posets $N C^{B}(n)$ of noncrossing partitions of type $B$ [17] and $N C^{B}(p, q)$ of annular noncrossing partitions, introduced and studied recently by Nica and Oancea [16]. Our main results are as follows. In Section 3 we prove that every interval of $B_{n}$ is shellable and present an example of a maximal element $x$ of $\operatorname{Abs}\left(D_{4}\right)$ for which the interval $[e, x]$ is not Cohen-Macaulay over any field (Example 3.3. In Section 4 we comment on the proof a $B_{n}$-analogue of [4, Theorem 1.1], stating that the order ideal $\mathcal{J}_{n}$ of $\operatorname{Abs}\left(B_{n}\right)$ generated by the set of Coxeter elements of $B_{n}$ is homotopy Cohen-Macaulay for all $n \geq 2$ (see Theorem 4.1). In particular, the order complex of $\mathcal{J}_{n} \backslash\{e\}$ is homotopy equivalent to a wedge of $(n-1)$-dimensional spheres and Cohen-Macaulay over $\mathbb{Z}$. The number of such spheres is also computed (see Theorem 4.8. We conjecture that the poset $\operatorname{Abs}\left(B_{n}\right)$ is Cohen-Macaulay for every $n \geq 2$ (i) Finally, in Section 5 we characterize the maximal intervals of $\operatorname{Abs}\left(B_{n}\right)$ and $\operatorname{Abs}\left(D_{n}\right)$ which are lattices and compute some of their enumerative invariants. We refer the reader to [18, Chapter 3] and [9, 19] for background on partially ordered sets and the topology of simplicial complexes, respectively.

(i) This conjecture has now been proved by the author 


\section{Preliminaries}

\subsection{The absolute length and absolute order}

Let $W$ be a finite Coxeter group with set of all reflections $\mathcal{T}$. Given $w \in W$, let $\ell_{\mathcal{T}}(w)$ denote the smallest integer $k$ such that $w$ can be written as a product of $k$ reflections in $\mathcal{T}$. The absolute order, or reflection length order, is the partial order on $W$ denoted by $\preceq$ and defined by letting

$$
u \preceq v \text { if and only if } \ell_{\mathcal{T}}(u)+\ell_{\mathcal{T}}\left(u^{-1} v\right)=\ell_{\mathcal{T}}(v)
$$

for $u, v \in W$. Equivalently, $\preceq$ is the partial order on $W$ with covering relations $w \rightarrow w t$, where $w \in W$ and $t \in \mathcal{T}$ are such that $\ell_{\mathcal{T}}(w)<\ell_{\mathcal{T}}(w t)$. In that case we write $w \stackrel{t}{\rightarrow} w t$. The poset $\operatorname{Abs}(W)$ is graded with rank function $\ell_{\mathcal{T}}$.

\subsection{The posets $\operatorname{Abs}\left(B_{n}\right)$ and $\operatorname{Abs}\left(D_{n}\right)$}

We view the hyperoctahedral group $B_{n}$ as the group of permutations $u$ of the set $\{ \pm 1, \pm 2, \ldots, \pm n\}$ such that $u(-i)=-u(i)$ for every $1 \leq i \leq n$. Following [11], the permutation which has cycle form $\left(a_{1} a_{2} \cdots a_{k}\right)\left(-a_{1}-a_{2} \cdots-a_{k}\right)$ is denoted by $\left(\left(a_{1}, a_{2}, \ldots, a_{k}\right)\right)$ and is called a paired $k$-cycle, while the cycle $\left(a_{1} a_{2} \cdots a_{k}-a_{1}-a_{2} \cdots-a_{k}\right)$ is denoted by $\left[a_{1}, a_{2}, \ldots, a_{k}\right]$ and is called a balanced $k$ cycle. Every element $u \in B_{n}$ can be written (uniquely) as a product of disjoint paired or balanced cycles, called cycles of $u$. With this notation, the set $\mathcal{T}$ of reflections of $B_{n}$ is equal to the union

$$
\{[i]: 1 \leq i \leq n\} \cup\{((i, j)),((i,-j)): 1 \leq i<j \leq n\} .
$$

The length $\ell_{\mathcal{T}}(u)$ of $u \in B_{n}$ is equal to $n-\gamma(u)$, where $\gamma(u)$ denotes the number of paired cycles in the cycle decomposition of $u$. An element $u \in B_{n}$ is maximal in $\operatorname{Abs}\left(B_{n}\right)$ if and only if it can be written as a product of disjoint balanced cycles whose lengths sum to $n$. The Coxeter elements of $B_{n}$ are precisely the balanced $n$-cycles. To simplify the notation, we will denote by $\ell$ the absolute length $\ell_{\mathcal{T}}$. The covering relations $w \stackrel{t}{\rightarrow} w t$ of $\operatorname{Abs}\left(B_{n}\right)$, when $w$ and $t$ are non-disjoint cycles, can be described as follows: for $1 \leq i<j \leq m \leq n$ we have:

(a) $\left(\left(a_{1}, \ldots, a_{i-1}, a_{i+1}, \ldots, a_{m}\right)\right) \stackrel{\left(\left(a_{i-1}, a_{i}\right)\right)}{\longrightarrow}\left(\left(a_{1}, \ldots, a_{m}\right)\right)$

(b) $\left(\left(a_{1}, \ldots, a_{m}\right)\right) \stackrel{\left[a_{i}\right]}{\longrightarrow}\left[a_{1}, \ldots, a_{i-1}, a_{i},-a_{i+1}, \ldots,-a_{m}\right]$

(c) $\left(\left(a_{1}, \ldots, a_{m}\right)\right) \stackrel{\left(\left(a_{i},-a_{j}\right)\right)}{\longrightarrow}\left[a_{1}, \ldots, a_{i},-a_{j+1}, \ldots,-a_{m}\right]\left[a_{i+1}, \ldots, a_{j}\right]$

(d) $\left[a_{1}, \ldots, a_{i-1}, a_{i+1}, \ldots, a_{m}\right] \stackrel{\left(\left(a_{i-1}, a_{i}\right)\right)}{\longrightarrow}\left[a_{1}, \ldots, a_{m}\right]$

(e) $\left[a_{1}, \ldots, a_{j}\right]\left(\left(a_{j+1}, \ldots, a_{m}\right)\right) \stackrel{\left(\left(a_{j}, a_{m}\right)\right)}{\longrightarrow}\left[a_{1}, \ldots, a_{m}\right]$

where $a_{1}, \ldots, a_{m}$ are elements of the set $\{ \pm 1, \ldots, \pm n\}$ with pairwise distinct absolute values.

The Coxeter group $D_{n}$ is the subgroup of index two of the group $B_{n}$ generated by the set of reflections

$$
\{((i, j)),((i,-j)): 1 \leq i<j \leq n\} .
$$


(these are all reflections in $D_{n}$ ). The absolute length on $D_{n}$ is the restriction of absolute length of $B_{n}$ on the set $D_{n}$. The number of balanced cycles of any element $u \in D_{n}$ is even and every Coxeter element of $D_{n}$ has the form $\left[a_{1}, a_{2}, \ldots, a_{n-1}\right]\left[a_{n}\right]$, where $\left|a_{i}\right| \in\{1,2, \ldots, n\}$ and $\left|a_{i}\right| \neq\left|a_{j}\right|$ for all $i \neq j$.

\section{Shellability}

In this section we prove the following theorem.

Theorem 3.1 Every interval of $A b s\left(B_{n}\right)$ is shellable.

Proof: (sketch) We show that every closed interval of $\operatorname{Abs}\left(B_{n}\right)$ admits an EL-labeling. The result then follows, since EL-shellability implies shellability (we refer to [8] for the definition of EL-labeling and EL-shellability).

Let $C\left(B_{n}\right)$ be the set of covering relations of $\operatorname{Abs}\left(B_{n}\right)$ and $(a, b) \in C\left(B_{n}\right)$. Then $a^{-1} b$ is a reflection of $B_{n}$, thus either $a^{-1} b=[i]$ for some $i \in\{1,2, \ldots, n\}$, or there exist $i, j \in\{1,2, \ldots, n\}$, with $i<j$, such that $a^{-1} b=((i, j))$ or $a^{-1} b=((i,-j))$. We define a map $\lambda: C\left(B_{n}\right) \rightarrow\{1,2, \ldots, n\}$ as follows:

$$
\lambda(a, b)= \begin{cases}i & \text { if } a^{-1} b=[i], \\ j & \text { if } a^{-1} b=((i, j)) \text { or }((i,-j)) .\end{cases}
$$

A similar labeling was used by Biane [6] in order to study the maximal chains of the poset $N C^{B}(n)$ of noncrossing $B_{n}$-partitions. Figure 1 illustrates the Hasse diagram of the interval $[e, x]$, for $n=4$ and $x=[3,-4]((1,2))$, together with the corresponding labels.

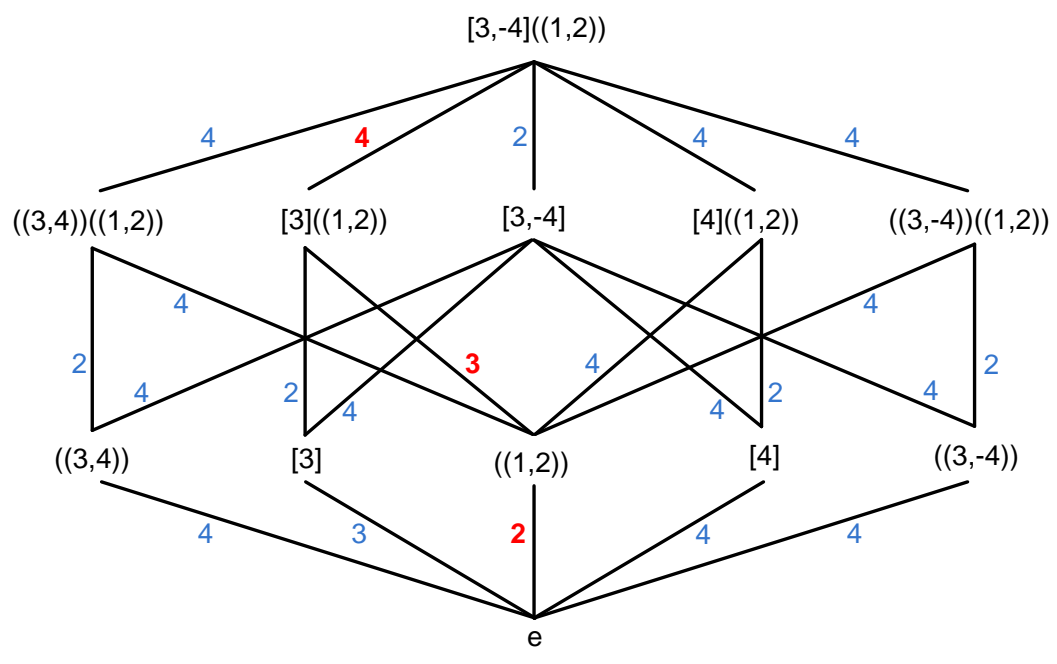

Fig. 1: The interval $[e, x]$ for $x=[3,-4]((1,2))$

The restricion of the map $\lambda$ to the interval $[x, y]$ is an EL-labeling for all $x, y \in B_{n}$ with $x \preceq y$. To prove that, it suffices to show that for every $u \in B_{n}$ the map $\left.\lambda\right|_{[e, u]}$ is an EL-labeling. Indeed, let 
$x, y \in B_{n}$ with $x \preceq y$ and define the map $\phi:[x, y] \rightarrow\left[e, x^{-1} y\right]$ by $\phi(t)=x^{-1} t$. Clearly, $\phi$ is a poset isomorphism. Moreover, if $(a, b) \in C([x, y])$, then $\phi(a)^{-1} \phi(b)=\left(x^{-1} a\right)^{-1} x^{-1} b=a^{-1} x x^{-1} b=a^{-1} b$, which implies that $\lambda(a, b)=\lambda(\phi(a), \phi(b))$.

Let $u=b_{1} b_{2} \cdots b_{k} p_{1} p_{2} \cdots p_{l}$ be written as a product of disjoint cycles, where $b_{i}=\left[b_{i}^{1}, \ldots, b_{i}^{k_{i}}\right]$ for $i \leq k$ and $p_{j}=\left(\left(p_{j}^{1}, \ldots, p_{j}^{l_{j}}\right)\right)$ with $p_{j}^{1}=\min \left\{\left|p_{j}^{m}\right|: 1 \leq m \leq l_{j}\right\}$ for $j \leq l$. We consider the sequence of positive integers obtained by placing the numbers $\left|b_{i}^{h}\right|$ and $\left|p_{j}^{m}\right|$, for $i, j, h \geq 1$ and $m>1$, in increasing order. There are $r=\ell(u)$ such integers. To simplify the notation, we denote by $c(u)=\left(c_{1}, c_{2}, \ldots, c_{r}\right)$ this sequence and say that $c_{\mu}(\mu=1,2, \ldots, r)$ belongs to a balanced (respectively paired) cycle if it is equal to some $\left|b_{i}^{h}\right|$ (respectively $\left|p_{j}^{m}\right|$ ). Clearly we have $c_{1}<c_{2}<\cdots<c_{r}$ and $\lambda(a, b) \in\left\{c_{1}, c_{2}, \ldots, c_{r}\right\}$ for every pair $a, b \in[e, u]$, with $a \rightarrow b$. To the sequence $\left(c_{1}, c_{2}, \ldots, c_{r}\right)$ corresponds a unique maximal chain

$$
\mathcal{C}_{u}: u_{0}=e \stackrel{c_{1}}{\rightarrow} u_{1} \stackrel{c_{2}}{\rightarrow} u_{2} \stackrel{c_{3}}{\rightarrow} \cdots \stackrel{c_{r}}{\rightarrow} u_{r}=u
$$

which can be constructed inductively as follows (here, the integer $\kappa$ in $a \stackrel{\kappa}{\rightarrow} b$ denotes the label $\lambda(a, b)$ ). If $c_{1}$ belongs to a balanced cycle, then $u_{1}=\left[c_{1}\right]$. Otherwise, if $c_{1}$ belongs to some $p_{i}$, say $p_{1}$, then we set $u_{1}$ to be either $\left(\left(p_{1}^{1}, c_{1}\right)\right)$ or $\left(\left(p_{1}^{1},-c_{1}\right)\right)$, so that $u_{1} \preceq p_{1}$ holds. In both cases $\lambda\left(e, u_{1}\right)=c_{1}$ and $\lambda\left(e, u_{1}\right)<\lambda(e, x)$ for any other atom $x \in[e, u]$. Suppose now that we have uniquely defined the elements $u_{1}, u_{2}, \ldots, u_{j}$, so that for every $i=1,2, \ldots, j$ we have $u_{i-1} \rightarrow u_{i}$ with $\lambda\left(u_{i-1}, u_{i}\right)=c_{i}$ and $\lambda\left(u_{i-1}, u_{i}\right)<\lambda\left(u_{i-1}, x\right)$ for every $x \in[e, u]$ such that $x \neq u_{i}$ and $u_{i-1} \rightarrow x$. We consider the number $c_{j+1}$ and distinguish two cases.

Case 1: $c_{j+1}$ belongs to a cycle whose elements have not been used. In this case, if $c_{j+1}$ belongs to a balanced cycle, then we set $u_{j+1}=u_{j}\left[c_{j+1}\right]$, while if $c_{j+1}$ belongs to $p_{s}$ for some $s \in\{1,2, \ldots, l\}$, then we set $u_{j+1}$ to be either $u_{j}\left(\left(p_{s}^{1}, c_{j+1}\right)\right)$ or $u_{j}\left(\left(p_{s}^{1},-c_{j+1}\right)\right)$, so that $u_{j}^{-1} u_{j+1} \preceq p_{s}$ holds.

Case 2: $c_{j+1}$ belongs to a cycle some element of which has been used. Then there exist an $i<j+1$ such that $c_{i}$ belongs to the same cycle as $c_{j+1}$. If $c_{i}, c_{j+1}$ belong to some $b_{s}$, then there is a balanced cycle of $u_{j}$, say $a$, that contains $c_{i}$. In this case we set $u_{j+1}$ to be the permutation that we obtain from $u_{j}$ if we add the number $c_{j+1}$ in the cycle $a$ in the same order and with the same sign that it appears in $b_{s}$. We proceed similarly if $c_{i}, c_{j+1}$ belong the same paired cycle.

Using the relations written in Section 2.2, one can show that $\mathcal{C}_{u}$ is lexicographically first and the unique strictly increasing chain in $[e, u]$. Thus Theorem 3.1 is proved.

Example 3.2 (i) Let $n=7$ and $u=[1,-7][3]((2,-6,-5))((4)) \in B_{7}$. Then $c(u)=(1,3,5,6,7)$ and $\mathcal{C}_{u}: e \stackrel{1}{\rightarrow}[1] \stackrel{3}{\rightarrow}[1][3] \stackrel{5}{\rightarrow}[1][3]((2,-5)) \stackrel{6}{\rightarrow}[1][3]((2,-6,-5)) \stackrel{7}{\rightarrow} u$.

(ii) Let $n=4$ and $v=[3,-4]((1,2))$. Then $c(v)=(2,3,4)$ and $\mathcal{C}_{v}: e \stackrel{2}{\rightarrow}((1,2)) \stackrel{3}{\rightarrow}((1,2))[3] \stackrel{4}{\rightarrow} v$.

Example 3.3 Figure 2 illustrates the Hasse diagram of the interval $I=[e, x]$ of $\operatorname{Abs}\left(D_{4}\right)$, where $x=$ $[1][2][3][4]$. Note that the Hasse diagram of the open interval $(e, x)$ is disconnected and, therefore, $I$ is not Cohen-Macaulay. It follows that $\operatorname{Abs}\left(D_{n}\right)$ is neither Cohen-Macaulay nor shellable for $n \geq 4$ [19. Corollary 3.1.9]. This is in accordance with Reiner's computations showing that $\operatorname{Abs}\left(D_{4}\right)$ is not CohenMacaulay and answers in the negative a question raised by Athanasiadis (personal communication), asking whether all intervals of the absolute order on a Coxeter group are shellable. 


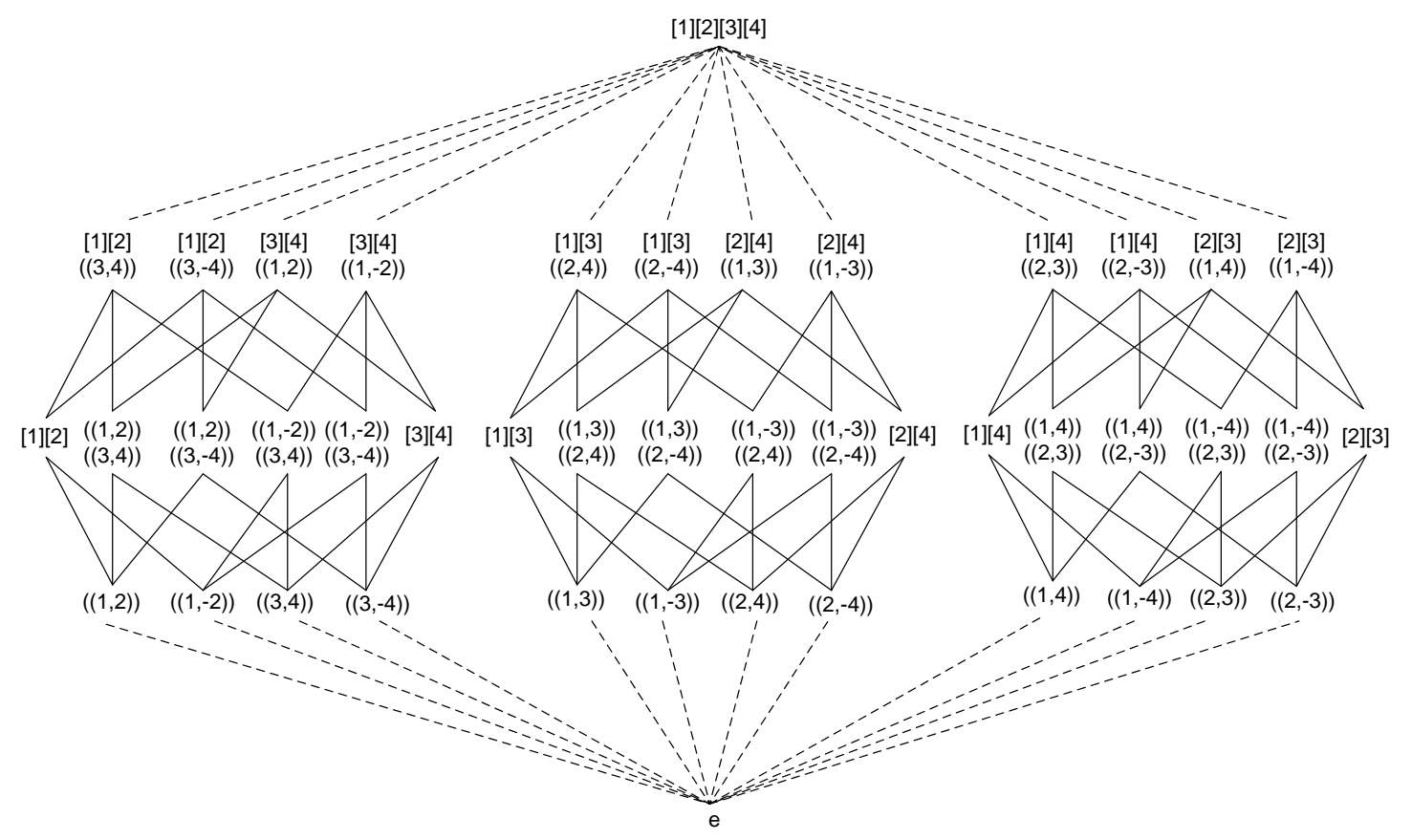

Fig. 2: The interval $[e,[1][2][3][4]]$ in $D_{4}$

\section{The ideal of Coxeter elements}

Recall that the Coxeter elements of $B_{n}$ are precisely the balanced $n$-cycles.

Theorem 4.1 The order ideal $\mathcal{J}_{n}$ of $A b s\left(B_{n}\right)$ generated by the set of Coxeter elements of $B_{n}$ is homotopy Cohen-Macaulay for all $n \geq 2$. In particular, the order complex of $\mathcal{J}_{n} \backslash\{e\}$ is homotopy equivalent to a wedge of $(n-1)$-dimensional spheres and Cohen-Macaulay over $\mathbb{Z}$.

Since the set of maximal elements of $\operatorname{Abs}\left(S_{n}\right)$ coincides with the set of Coxeter elements of $S_{n}$, Theorem 4 can be considered as a $B_{n}$-analogue of [4, Theorem 1.1]. It is not known whether the order ideal generated by the Coxeter elements is Cohen-Macaulay for every Coxeter group $W$. To prove Theorem 4.1 we will use the notion of strong constructibility, introduced in [4]. We first review some definitions and results given in [4].

Definition 4.2 $A d$-dimensional simplicial complex $\Delta$ is constructible if it is a simplex or it can be written as $\Delta=\Delta_{1} \cup \Delta_{2}$, where $\Delta_{1}, \Delta_{2}$ are d-dimensional constructible simplicial complexes such that $\Delta_{1} \cap \Delta_{2}$ is constructible of dimension at least $d-1$.

We do not know whether this notion of constructibility coincides with the classical notion, which differs in that the dimension of the intersection $\Delta_{1} \cap \Delta_{2}$ has to equal to $d-1$. However, it is proved in [4] that every constructible simplicial complex, in the sense of Definition 4.2, is homotopy Cohen-Macaulay. Figure 3 illustrates two 2-dimensional strongly constructible complexes, $\Delta_{1}$ and $\Delta_{2}$, the intersection of which is the 2-dimensional simplex $F_{3}$. Thus, the union $\Delta_{1} \cup \Delta_{2}$ is strongly constructible as well. 


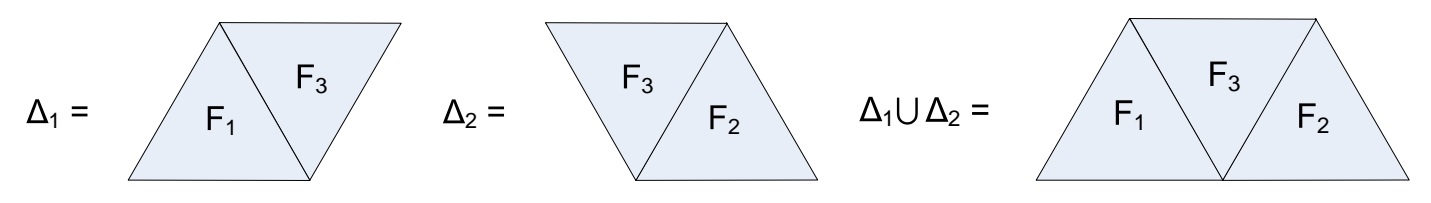

Fig. 3: A 2-dimensional strongly constructible simplicial complex

Definition 4.3 A finite poset $P$ of rank $d$ with a minimum element is strongly constructible if it is bounded and pure shellable or it can be written as a union $P=I_{1} \cup I_{2}$ of two strongly constructible proper ideals $I_{1}, I_{2}$ of rank $d$, such that $I_{1} \cap I_{2}$ is strongly constructible of rank at least $d-1$.

Proposition 4.4 The order complex of any strongly constructible poset is constructible.

Remark 4.5 Every strongly constructible poset is homotopy Cohen-Macaulay.

Proposition 4.6 The poset $\operatorname{Abs}\left(S_{n}\right)$ is strongly constructible for every $n \geq 1$.

The main idea to prove Proposition 4.6 is to partition the set of maximal elements (n-cycles) of $\operatorname{Abs}\left(S_{n}\right)$ by placing $x$ and $y$ in the same part of the partition if $x(1)=y(1)$. This is the partition of $S_{n}$ into the left cosets of the subgroup which consists of the permutations of the set $\{2,3, \ldots, n\}$. Then we show that the order ideal generated by each part is strongly constructible and that so is the intersection of two or more of these ideals. We extend this construction to the case of $\mathcal{J}_{n} \subset B_{n}$ by defining the following equivalence relation on the set of cycles of $B_{n}$.

Definition 4.7 Given cycles $u, v$ of $B_{n}$, we write $u \sim v$ if

- $u, v$ are either both paired or both balanced cycles and

- $u(i)= \pm v(i)$ for every $i=1,2, \ldots, n$.

We denote by $\bar{u}$ the equivalence class of $u \in B_{n}$. If $u_{1}, u_{2}, \ldots, u_{k}$ are disjoint cycles of $B_{n}$, we set $\bar{u}_{1} \bar{u}_{2} \cdots \bar{u}_{k}=\left\{v_{1} v_{2} \cdots v_{k}: v_{i} \in \bar{u}_{i}, i=1,2, \ldots, k\right\} \subset B_{n}$. For example,

$$
\overline{((1,2))} \overline{[3,4]}=\{((1,2))[3,4],((1,-2))[3,4],((1,2))[3,-4],((1,-2))[3,-4]\} \text {. }
$$

Let $\alpha=\left(\alpha_{1}, \alpha_{2}, \ldots, \alpha_{k}\right)$ be a sequence of distinct positive integers, with $\alpha_{i} \leq n$ for every $i=$ $1,2, \ldots, n$. To the sequence $\alpha$ we associate the permutations $[\alpha]=\left[\alpha_{1}, \alpha_{2}, \ldots, \alpha_{k}\right]$ and $((\alpha))=$ $\left(\left(\alpha_{1}, \alpha_{2}, \ldots, \alpha_{k}\right)\right)$ of $B_{n}$ and the cycle $(\alpha)=\left(\alpha_{1} \alpha_{2} \cdots \alpha_{k}\right)$ of $S_{n}$. Let $A$ be a subset of $S_{n}$ consisting of permutations that have length equal to $n-k$ (i.e. permutations of $S_{n}$ that have exactly $k$ cycles in their decomposition). To the order ideal $\langle A\rangle$ of $\operatorname{Abs}\left(S_{n}\right)$, which has rank $n-k$, we associate the order ideal $\overline{\langle A\rangle}$ of $\operatorname{Abs}\left(B_{n}\right)$, which has rank $n-k+1$ and is defined as:

$$
\overline{\langle A\rangle}:=\left\langle x \in \overline{\left[\alpha_{1}\right]} \overline{\left(\left(\alpha_{2}\right)\right)} \overline{\left(\left(\alpha_{3}\right)\right)} \cdots \overline{\left(\left(\alpha_{k}\right)\right)}:\left(\alpha_{1}\right)\left(\alpha_{2}\right)\left(\alpha_{3}\right) \cdots\left(\alpha_{k}\right) \in A\right\rangle .
$$

Let $A_{1}, A_{2}$ be subsets of $S_{n}$ as above. Then $\overline{\left\langle A_{1}\right\rangle} \cup \overline{\left\langle A_{2}\right\rangle}=\overline{\left\langle A_{1} \cup A_{2}\right\rangle}$ and $\overline{\left\langle A_{1}\right\rangle} \cap \overline{\left\langle A_{2}\right\rangle}=$

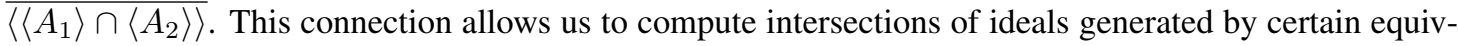
alence classes, using the intersections of ideals generated by the corresponding cycles in $S_{n}$ and to adapt the proof of [4, Proposition 4.2].

The next result is a $B_{n}$-analogue of [4, Theorem 1.2]. 
Theorem 4.8 Let $\mathcal{J}_{n}$ denote the order ideal of $A b s\left(B_{n}\right)$ generated by the Coxeter elements of $B_{n}$ and $\overline{\mathcal{J}}_{n}=\mathcal{J}_{n} \backslash\{\hat{0}\}$. The reduced Euler characteristic of the order complex $\Delta\left(\overline{\mathcal{J}}_{n}\right)$ satisfies

$$
\sum_{n \geq 2}(-1)^{n} \tilde{\chi}\left(\Delta\left(\overline{\mathcal{J}}_{n}\right)\right) \frac{t^{n}}{n !}=1-\sqrt{C(2 t)} \exp \{-2 t C(2 t)\}\left(1+\sum_{n \geq 1} 2^{n-1}\left(\begin{array}{c}
2 n-1 \\
n
\end{array}\right) \frac{t^{n}}{n}\right)
$$

where $C(t)=\frac{1}{2 t}(1-\sqrt{1-4 t})$ is the ordinary generating function for the Catalan numbers.

\section{Combinatorics of intervals}

\subsection{Intervals with the lattice property}

In this section we characterize the maximal intervals in $\operatorname{Abs}\left(B_{n}\right)$ and $\operatorname{Abs}\left(D_{n}\right)$ which are lattices. It is known that the interval $[e, c]$ of $\operatorname{Abs}(W)$ is a lattice for every finite Coxeter group $W$ and Coxeter element $c$ of $W$ (see [5, Fact 2.3.1], [11, Section 4], [12]). Moreover, it was shown in [16, Theorem 1.6] that $[e, x]$ is a lattice for every maximal element $x$ of $\operatorname{Abs}\left(B_{n}\right)$ that is a product of exactly two Coxeter elements, one of which is a reflection.

Theorem 5.1 Let $x$ be a maximal element of $A b s\left(B_{n}\right)$. The interval $[e, x]$ of $A b s\left(B_{n}\right)$ is a lattice if and only if $x$ has the form

$$
x=\left[a_{1}, a_{2}, \ldots, a_{k}\right]\left[a_{k+1}\right]\left[a_{k+2}\right] \cdots\left[a_{n}\right]
$$

where $k \in\{0,1, \ldots, n\}$ and the $a_{i} \in\{ \pm 1, \pm 2, \ldots, \pm n\}$ have pairwise disjoint absolute values.

We now consider the absolute order on the group $D_{n}$ and prove the following theorem.

Theorem 5.2 Let $x$ be a maximal element of $A b s\left(D_{n}\right)$. The interval $[e, x]$ of $A b s\left(D_{n}\right)$ is a lattice if and only if $x$ is a Coxeter element or $n=4$ and $x=[1][2][3][4]$.

Proof: As previously mentioned, the interval $[e, x]$ of $\operatorname{Abs}\left(D_{n}\right)$, where $x$ ia a Coxeter element of $D_{n}$, is known to be a lattice. Let $x$ be a maximal non-Coxeter element of $\operatorname{Abs}\left(D_{n}\right)$ such that the interval $[e, x]$ of $\operatorname{Abs}\left(D_{n}\right)$ is a lattice. One can show that in this case at most one cycle of $x$ is not a reflection. Thus we may write $x=\left[a_{1}, a_{2}, \ldots, a_{m}\right]\left[b_{2}\right] \cdots\left[b_{k}\right]$, where $k>2$ and $m+k-1=n$. Suppose that $m \geq 2$. Then $u=\left[a_{1}, a_{2}\right]\left[b_{2}\right]$ and $v=\left[a_{1}, a_{2}\right]\left[b_{3}\right]$ are elements of $[e, x]$. However, the intersection $[e, u] \cap[e, v] \subset$ $\operatorname{Abs}\left(D_{n}\right)$ has two maximal elements, namely the paired reflections $\left(\left(a_{1}, a_{2}\right)\right)$ and $\left(\left(a_{1},-a_{2}\right)\right)$. This implies that the elements $u$ and $v$ do not have a meet in $[e, x]$ and, therefore, the interval $[e, x]$ is not a lattice. Thus we must have $m=1$, so $k=n$ and $x=[1][2] \cdots[n]$. Suppose that $n \geq 5$. We consider the elements $u=[1][2][3][4]$ and $v=[1][2][3][5]$ of $[e, x]$ and note that the intersection $[e, u] \cap[e, v]$ has three maximal elements, namely $[1][2],[1][3]$ and $[2][3]$. This implies that the interval $[e, x]$ is not a lattice, contradicting our assumption. Thus $n=4$ and $x=[1][2][3][4]$. Figure 2 shows that the interval $[e,[1][2][3][4]]$ is indeed a lattice and Theorem 5.2 is proved.

Remark 5.3 For the remainder of this paper we denote by $L(k, r)$ the order ideal of $\operatorname{Abs}\left(B_{n}\right)$ generated by the element $[1,2, \ldots, k][k+1] \cdots[k+r]$, where $k, r$ are nonnegative integers such that $k+r \leq n$. Moreover, we set $L_{n}:=L(0, n)=[e,[1][2] \cdots[n]]$. 


\subsection{The lattice $L_{n}$}

We compute some of the basic enumerative invariants of the lattice $L_{n}$, as follows.

Proposition 5.4 For the lattice $L_{n}$ the following hold:

(i) The number of elements of $L_{n}$ is equal to

$$
\sum_{k=0}^{\lfloor n / 2\rfloor}\left(\begin{array}{c}
n \\
2 k
\end{array}\right) 2^{n-k}(2 k-1) ! !
$$

(ii) The number of elements of $L_{n}$ of rank $r$ is equal to

$$
\sum_{k=0}^{\min \{r, n-r\}} \frac{n !}{k !(r-k) !(n-r-k) !} .
$$

(iii) The zeta polynomial of $L_{n}$ is given by the formula

$$
Z_{n}(m)=\sum_{k=0}^{\lfloor n / 2\rfloor}\left(\begin{array}{c}
n \\
2 k
\end{array}\right) m^{n-k}(m-1)^{k}(2 k-1) ! ! .
$$

(iv) The number of maximal chains of $L_{n}$ is equal to

$$
n ! \sum_{k=0}^{\lfloor n / 2\rfloor}\left(\begin{array}{c}
n \\
2 k
\end{array}\right)(2 k-1) ! !
$$

(v) For the Möbius function of $L_{n}$ we have

$$
\mu_{n}(\hat{0}, \hat{1})=(-1)^{n} \sum_{k=0}^{\lfloor n / 2\rfloor}\left(\begin{array}{c}
n \\
2 k
\end{array}\right) 2^{k}(2 k-1) ! !,
$$

where $\hat{0}$ and $\hat{1}$ denotes the minimum and the maximum element of $L_{n}$, respectively.

Remark 5.5 By the proof of Theorem 3.1, the lattice $L_{n}$ is EL-shellable. We describe two more ELlabelings for $L_{n}$.

- Let $\Lambda=\{[i]: i=1,2, \ldots, n\} \cup\{((i, j)): 1 \leq i<j \leq n\}$. We linearly order the elements of $\Lambda$ in the following way. We first order the balanced reflections so that $[i]<_{\Lambda}[j]$ if and only if $i<j$. Then we order the paired reflections lexicographically. Finally, we set $[n]<_{\Lambda}((1,2))$. The map $\lambda_{1}: C\left(B_{n}\right) \rightarrow \Lambda$ defined as

$$
\lambda_{1}(a, b)= \begin{cases}{[i]} & \text { if } a^{-1} b=[i], \\ ((i, j)) & \text { if } a^{-1} b=((i, j)) \text { or }((i,-j))\end{cases}
$$

is an EL-labeling for $L_{n}$. 
- Let $\mathcal{T}$ be the set of reflections of $B_{n}$. We define a total order $<_{\mathcal{T}}$ on $\mathcal{T}$ which extends the order $<_{\Lambda}$, by ordering the reflections $((i,-j))$, for $1 \leq i<j \leq n$, lexicographically and letting $((n-1, n))<\mathcal{T}$ $((1,-2))$. Let $t_{i}$ be the $i$-th reflection in the order above. We define a map $\lambda_{2}: C\left(B_{n}\right) \rightarrow\left\{1,2, \ldots, n^{2}\right\}$ as

$$
\lambda_{2}(a, b)=\min _{1 \leq i \leq n^{2}}\left\{i: t_{i} \vee a=b\right\},
$$

where $t_{i} \vee a$ denotes the join of $t_{i}$ and $a$ in the lattice $L_{n}$. The map $\lambda_{2}$ is an EL-labeling for $L_{n}$.

See Figure 4 for an example of these two EL-labelings when $n=2$.
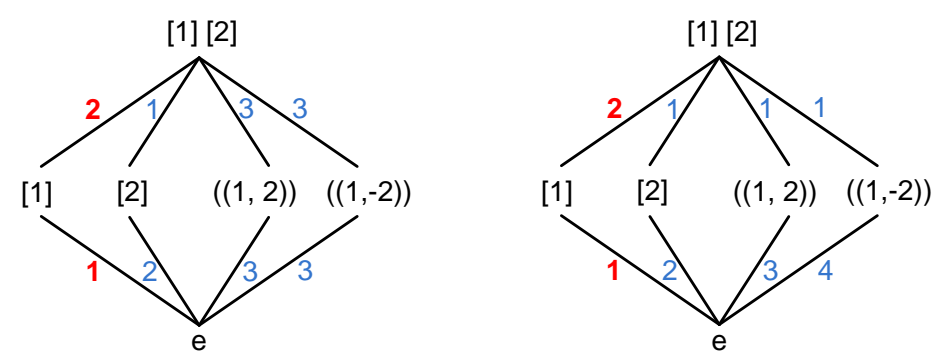

Fig. 4: EL-labelings for $L_{2}$

\subsection{Enumerative combinatorics of $L(k, r)$}

In this section we compute the cardinality, zeta polynomial and Möbius function of $L(k, r)$, where $k, r$ are nonnegative integers with $k+r=n$. The case $k=n-1$ was treated by Goulden, Nica and Oancea [14]. We will use their results, as well as the formulas for the cardinality and zeta polynomial for $N C^{B}(n)$ and Proposition 5.4, to find the corresponding formulas for $L(k, r)$.

Proposition 5.6 Let $\alpha_{r}=\left|L_{r}\right|, \beta_{r}(m)=Z\left(L_{r}, m\right)$ and $\mu_{r}=\mu_{r}\left(L_{r}\right)$, where $\alpha_{r}=\beta_{r}(m)=\mu_{r}=1$ for $r \in\{0,1\}$. For fixed nonnegative integers $k, r$ such that $k+r=n$, the cardinality, zeta polynomial and Möbius function of the lattice

$$
L(k, r)=[e,[1,2, \ldots, k][k+1] \cdots[k+r]]
$$

are given by:

- $\# L(k, r)=\left(\begin{array}{c}2 k \\ k\end{array}\right)\left(\frac{2 r k}{k+1} \alpha_{r-1}+a_{r}\right)$,

- $Z(L(k, r), m)=\left(\begin{array}{c}m k \\ k\end{array}\right)\left(\frac{2 r k}{k+1}(m-1) \beta_{r-1}(m)+\beta_{r}(m)\right)$,

- $\mu(L(k, r))=(-1)^{n}\left(\begin{array}{c}2 k-1 \\ k\end{array}\right)\left(\frac{4 r k}{k+1}\left|\mu_{r-1}\right|+\left|\mu_{r}\right|\right)$. 
Proof: (sketch) We denote by $A$ the subset of $L(k, r)$ which consists of elements $x$ with the following property: every cycle of $x$ that contains at least one of $\pm 1, \pm 2, \ldots, \pm k$ is less than or equal to the element $[1,2, \ldots, k]$ in $\operatorname{Abs}\left(B_{n}\right)$. Let $x=x_{1} x_{2} \cdots x_{\nu} \in A$, written as a product of disjoint cycles. Without loss of generality, we may assume that there is a $t \in\{0,1, \ldots, \nu\}$ such that $x_{1} x_{2} \cdots x_{t} \preceq[1,2, \ldots, k]$ and $x_{t+1} x_{t+2} \cdots x_{\nu} \preceq[k+1][k+2] \cdots[k+r]$. Clearly, there exist a poset isomorphism

$$
\begin{aligned}
f: A & \rightarrow N C^{B}(k) \times[e,[k+1] \cdots[k+r]] \\
x & \mapsto\left(x_{1} \cdots x_{t}, \quad x_{t+1} \cdots x_{\nu}\right),
\end{aligned}
$$

so that

$$
A \cong N C^{B}(k) \times L_{r}
$$

Let $C:=L(k, r) \backslash A$ and $x=x_{1} x_{2} \cdots x_{\nu} \in C$, written as a product of disjoint cycles. Then there exists a paired cycle of $x$, say $x_{1}$, and a reflection $((i, j))$ with $|j| \in\{1,2, \ldots, k\}, j \in\{k+1, k+2, \ldots, k+r\}$, such that $((i, j)) \preceq x_{1}$. Note that the cycle $x_{1}$ and the reflection $((i, j))$ are unique with this property. For every $j \in\{k+1, k+2, \ldots, k+r\}$ denote by $C_{j}$ the set of permutations $x \in L(k, r)$ which have a cycle, say $x_{1}$, such that $((i, j)) \preceq x_{1}$ for some $i \in\{ \pm 1, \pm 2, \ldots, \pm k\}$. Thus, for every $x \in C$ there exists an ordering $x_{1}, x_{2}, \ldots, x_{\nu}$ of the cycles of $x$ and a unique index $t \in\{1,2, \ldots, \nu\}$ such that $x_{1} x_{2} \cdots x_{t} \preceq[1,2, \ldots, k][j]$ and $x_{t+1} x_{t+2} \cdots x_{\nu} \preceq[k+1][k+2] \cdots[j-1][j+1] \cdots[k+r]$. Let

$$
E_{j}=\{x \in C: x \preceq[1,2, \ldots, k][j]\} .
$$

Clearly, there exist a poset isomorphism

$$
\begin{aligned}
g_{j}: C_{j} & \rightarrow E_{j} \quad \times[e,[k+1] \cdots[j-1][j+1] \cdots[k+r]] \\
x & \mapsto\left(x_{1} \cdots x_{t} \quad, \quad x_{t+1} \cdots x_{\nu}\right),
\end{aligned}
$$

so that

$$
C_{j} \cong E_{l} \times L(0, r-1) .
$$

The results follow by using the poset isomorphisms (3) and (4) and [14, Section 5].

\section{Acknowledgments}

I am grateful to Christos Athanasiadis for valuable conversations, for his encouragement and for his careful reading and comments on preliminary versions of this paper. I would also like to thank Christian Krattenthaler, Victor Reiner and Volkmar Welker for helpful discussions.

\section{References}

[1] D. Armstrong, Braid groups, clusters and free probability: an outline from the AIM Workshop, January 2005, available at http: / / wWW . a imath. org/WWN/braidgroups /.

[2] D. Armstrong, Generalized noncrossing partitions and combinatorics of Coxeter groups, preprint, 2007, math. CO/ 0611106 , Mem. Amer. Math. Soc. (to appear).

[3] C.A. Athanasiadis, T. Brady and C. Watt, Shellability of noncrossing partition lattices, Proc. Amer. Math. Society 135 (2007), 939-949. 
[4] C.A. Athanasiadis and M. Kallipoliti, The absolute order on the symmetric group, constructible partially ordered sets and Cohen-Macaulay complexes, J. Combin. Theory Series A 115 (2008), 1286-1295.

[5] D. Bessis, The dual braid monoid, Ann. Sci. Ecole Norm. Sup. 36 (2003), 647-683.

[6] P. Biane, Parking functions of types A and B, Electron. J. Combin. 9 (2002), Note 7, 5 pp (electronic).

[7] A. Björner, Orderings of Coxeter groups, in Combinatorics and Algebra, Boulder 1983 (C. Greene, ed.), Contemp. Math. 34, Amer. Math. Society, Providence, RI, 1984, pp. 175-195

[8] A. Björner, Shellable and Cohen-Macaulay partially ordered sets, Trans. Amer. Math. Soc. 260 (1980), 159-183.

[9] A. Björner, Topological methods, in Handbook of combinatorics (R.L. Graham, M. Grötschel and L. Lovász, eds.), North Holland, Amsterdam, 1995, pp. 1819-1872.

[10] A. Björner and F. Brenti, Combinatorics of Coxeter groups, Graduate Texts in Mathematics 231, Springer-Verlag, New York, 2005.

[11] T. Brady and C. Watt, $K(\pi, 1)$ 's for Artin groups of finite type, in Proceedings of the Conference on Geometric and Combinatorial group theory, Part I (Haifa 2000), Geom. Dedicata 94 (2002), $225-250$.

[12] T. Brady and C. Watt, Non-crossing partition lattices in finite real reflection groups, Trans. Amer. Math. Soc. 360 (2008), 1983-2005.

[13] P. Diaconis and R.L. Graham, Spearman's footrule as a measure of disarray, J. Roy. Statist. Soc. Ser. B 39 (1977), 262-268.

[14] I.P. Goulden, A. Nica and I. Oancea, Enumerative properties of $N C^{B}(p, q)$, preprint, 2007, math.CO/0708.2212, Ann. Comb. (to appear).

[15] J.E. Humphreys, Reflection groups and Coxeter groups, Cambridge Studies in Advanced Mathematics 29, Cambridge University Press, Cambridge, England, 1990.

[16] A. Nica and I. Oancea, Posets of annular noncrossing partitions of types $B$ and D, Discrete Math. 309 (2009), 1443-1466.

[17] V. Reiner, Non-crossing partitions for classical reflection groups Discrete Math. 177 (1997), 195222.

[18] R.P. Stanley, Enumerative Combinatorics, vol. 1, Wadsworth \& Brooks/Cole, Pacific Grove, CA, 1986; second printing, Cambridge Studies in Advanced Mathematics 49, Cambridge University Press, Cambridge, 1997.

[19] M. Wachs, Poset Topology: Tools and Applications, in Geometric Combinatorics (E. Miller, V. Reiner and B. Sturmfels, eds.), IAS/Park City Mathematics Series 13, pp. 497-615, Amer. Math. Society, Providence, RI, 2007. 